\title{
Factibilidad técnica del uso de escorias de cobre como reemplazo de arena en morteros de pega de muros de albañileria
}

Technical feasibility of the use of copper slag as sand replacement in mortars of masonry walls

Mauricio Pradena-Miquel ${ }^{1}$, Patricio Cendoya-Hernández², Andrés Borkowsky-Opazo ${ }^{3}$

Fecha de recepción: 20 de junio de 2018

Fecha de aprobación: 5 de setiembre de 2018

Pradena-Miquel, M. Factibilidad técnica del uso de escorias de cobre como reemplazo de arena en morteros de pega de muros de albañileria. Tecnología en Marcha. Vol. 32-2. AbrilJunio 2019. Pág 100-111.

DOI: https://doi.org/10.18845/tm.v32i2.4360

1 Prof. Asistente, Departamento de Ingeniería Civil, Universidad de Concepción, Chile. Correo electrónico: mpradena@udec.cl

2 Prof. Asociado. Departamento de Ingeniería Civil, Universidad de Concepción, Chile. Correo electrónico: pcendoya@udec.cl

3 Ingeniero Civil. Departamento de Ingeniería Civil, Universidad de Concepción, Chile. Correo electrónico: aborkowsky@udec.cl 


\title{
Palabras clave
}

Morteros; Escorias de cobre; Muros de albañilería; Sustentabilidad.

\begin{abstract}
Resumen
Chile es uno de los mayores generadores de escorias de fundición de cobre (EFC) a nivel mundial con alrededor de 50 millones de toneladas acumuladas. Debido a que las EFC poseen características fisicoquímicas similares a las materias primas del cemento, resulta atractivo utilizar la EFC para la producción de morteros de pega sustentables para su uso en muros de albañilería. De esta manera el objetivo del presente artículo es estudiar la factibilidad técnica de morteros de pega fabricados con un árido fino obtenido a partir de la combinación de arena y granalla de EFC para su utilización en muros de albañilería confinada. Esto se realiza comparando en laboratorio sus propiedades y características con respecto a un mortero patrón (sin EFC). Se analizan propiedades del mortero de pega, tales como la consistencia, densidad, resistencia a la compresión, resistencia a la flexión y resistencia a la adherencia; además de su influencia en la resistencia a la compresión y al corte de muros de albañilería confinada. Los resultados muestran que la incorporación de la EFC en morteros de pega para muros de albañilería tiene una influencia positiva, ya que se incrementan significativamente las resistencias de adherencia $(20,2 \%)$, resistencia a la compresión $(18,1 \%)$ y resistencia al corte (12\%). De esta manera, se concluye que para las condiciones analizadas, utilizar de morteros de pega con EFC en muros de albañilería confinada es la técnicamente factible.
\end{abstract}

\section{Keywords}

Mortars; Copper slags; Masonry walls; Sustainability.

\begin{abstract}
Chile is one of the largest generators of copper slags (CS) worldwide with around 50 million tons accumulated. As the CS have similar physicochemical characteristics to the raw materials of cement, their application in the production of sustainable mortars for use in masonry walls is very attractive. The objective of the present article is to study the technical feasibility of the use of CS as sand replacement in cement mortars of masonry walls. For that, their properties and characteristics are compared in laboratory with a standard mortar (without CS). Different properties of the cement mortar are analyzed, such as consistency, density, flexural and compressive strength, and adhesive strength. Moreover, its influence on the compressive and shear strength of confined masonry elements is also analyzed. The results show that the incorporation of CS in masonry mortars has a positive influence, increasing the adhesive strength $(20.2 \%)$, compressive strength (18.1\%) and shear strength (12\%). Therefore, for the conditions analyzed, the use of cement mortars with CS in confined masonry walls is technically feasible.
\end{abstract}

\section{Introducción}

Chile al ser es uno de los mayores productores de cobre a nivel mundial genera grandes volúmenes de residuos, desechos y subproductos, entre los que se encuentran las escorias de fundición de cobre (EFC), las cuales constituyen uno de los principales desechos sólidos en las plantas pirometalúrgicas de la industria del cobre. Se calcula que por cada tonelada de cobre producido se generan alrededor de 2.2 toneladas de escorias, y tan solo en la división El Teniente de Codelco, se producen cerca de 700.000 toneladas de escoria al año. Se estima que 
Chile posee un volumen histórico acumulado de aproximadamente 50 millones de toneladas [1], las que son catalogadas como pasivos ambientales que son potencialmente contaminantes si no son bien manejados. Además, los volúmenes vertidos ocupan grandes extensiones de superficies que muchas veces restan terreno cultivable y producen contaminación visual del paisaje. Es por ello que surge la necesidad de encontrar usos alternativos a las escorias, de modo de reutilizar y dar un valor agregado a lo que se genera como subproducto de las fundiciones de cobre.

Por otra parte, se conoce que las escorias poseen en su composición química óxidos de silicio, aluminio, hierro y calcio, los que se encuentran de igual manera en la estructura química del cemento, por lo que la interacción entre ambos compuestos es viable químicamente. Además, al ser procesadas adquieren características similares a la de los áridos finos con forma, granulometría y textura definidas, las cuales permiten que puedan ser utilizadas como reemplazo parcial o total del agregado fino presente en morteros y hormigones. Por todo lo anterior resulta atractiva la utilización de EFC para la producción de morteros sustentables.

Diversos investigadores [1-7] han estudiado el comportamiento y el efecto de incluir EFC como agregado en morteros y hormigones. Moura y Coutinho [2] investigaron la resistencia a la compresión y flexotraccion de hormigones con porcentajes de reemplazo del árido fino del $30 \%, 40 \%$ y $50 \%$ para distintas razones Agua/Cemento (A/C). Los resultados indicaron que el hormigón con un porcentaje de reemplazo del 40\% de EFC, incrementa la resistencia a la flexotraccion en un $10 \%$ con respecto al mortero de referencia, además el peso específico se ve incrementado en el orden de un 10\% con respecto al hormigón de referencia cuando utiliza un 50\% de EFC como reemplazo. Hwang y Laiw [3] evaluaron la resistencia a la compresión desarrollada en morteros y hormigones con contenidos distintos de EFC como agregado fino para diferentes relaciones $\mathrm{A} / \mathrm{C}$. Las resistencias de las mezclas con porcentajes del $20-80 \%$ de reemplazo de EFC fueron superiores a las muestras de referencia. Li [4] y Zong [5] también reportaron que hormigones con contenidos de EFC como reemplazo parcial del árido fino, poseen propiedades mecánicas similares a los que contienen áridos convencionales. Al-Jabri [6] confeccionó mezclas de hormigón con distintas proporciones de EFC como reemplazo parcial y total del agregado fino. Los resultados muestran aumentos de densidad y trabajabilidad con respecto a hormigones de referencia, y aumento de las resistencias a compresión y flexotraccion para algunos porcentajes de reemplazos. Cares [7] fabricó hormigones con EFC como reemplazo parcial del árido fino en distintos porcentajes (25, 40 y 50\%) y distintas razones $\mathrm{A} / \mathrm{C}$, analizando su comportamiento a través de la resistencia a compresión y flexotracción. Los resultados indican que la adición de EFC mejora significativamente las resistencias de estos, con respecto a los morteros de referencia, obteniendo el mayor desempeño para el $40 \%$ de reemplazo [1].

El objetivo del presente artículo es estudiar la factibilidad técnica de morteros de pega fabricados con un árido fino obtenido a partir de la combinación de arena Bío-Bío y granalla de EFC en proporción del 40\% de remplazo, para su utilización en muros de albañilería confinada.

\section{Materiales y metodos}

\section{Materiales}

Para la confección del mortero se utilizaron dos áridos finos, la arena Bío-Bío y la granalla de EFC. Esta última posee una textura lisa y poco porosa, forma granular, cubica y angulosa, y con granulometría entre 0.3 y $2.5 \mathrm{~mm}$ (figura 1 y 2). Las propiedades físicas más importantes de los áridos finos se resumen en el cuadro 1. 
Un análisis granulométrico de los áridos finos disponibles, indicó que estos no cumplen con la banda granulométrica de la NCh163 Of. 79 [8], específicamente en la última fracción de la banda por falta de porcentaje de finos. En el caso de la EFC en los tamices $\mathrm{N}^{\circ} 30$ y $\mathrm{N}^{\circ} 50$ y de la arena Bío-Bío en los tamices №50 y N¹00, respectivamente. Sin embargo, este último se considera aceptable debido a que los porcentajes son muy cercanos a los límites y el material posee un uso masivo en la zona y un buen comportamiento en hormigones y morteros fabricados.

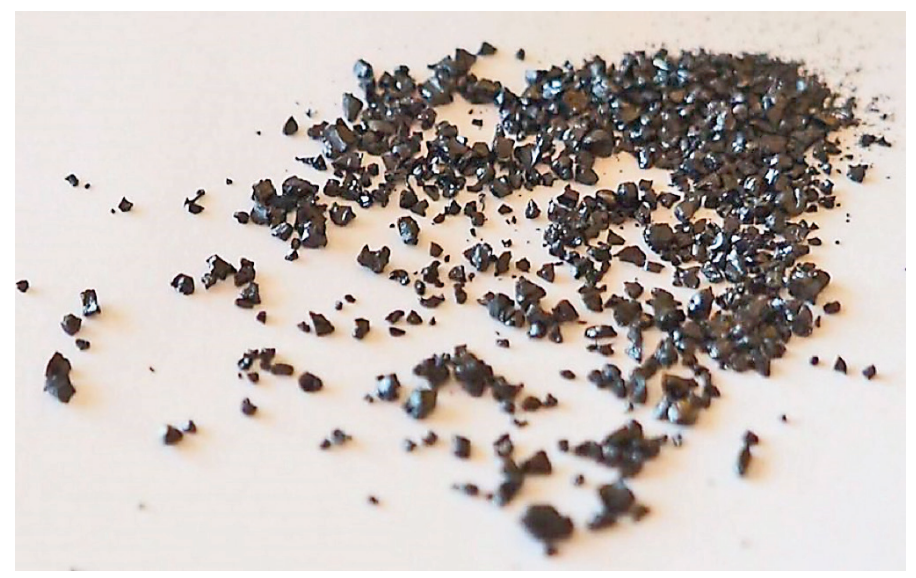

Figura 1. Granalla de EFC.

Cuadro 1. Propiedades físicas de la EFC y la arena Bío-Bío.

\begin{tabular}{|c|c|c|c|}
\hline Constantes Físicas & Arena & EFC & Unidad \\
\hline Densidad aparente suelta seca & 1530 & 2050 & $\mathrm{Kg} / \mathrm{m} 3$ \\
\hline Densidad aparente compacta seca & 1610 & 2200 & $\mathrm{Kg} / \mathrm{m} 3$ \\
\hline Densidad real seca & 2700 & 3800 & $\mathrm{Kg} / \mathrm{m} 3$ \\
\hline Absorción & 1.6 & 0.15 & $\%$ \\
\hline Porcentaje de huecos compactados & 43.33 & 46.05 & $\%$ \\
\hline Fino con lavado bajo malla 200 A.S.T.M & 0.1 & 0.2 & $\%$ \\
\hline Módulo de finura & 2.82 & 3.28 & - \\
\hline Impurezas orgánicas según A.S.T.M & No contiene & No contiene & - \\
\hline
\end{tabular}

Al combinar estos áridos en proporciones en volumen de 40\% EFC y 60\% arena, la curva granulométrica se ajusta de mejor manera que la de la EFC o arena por sí sola, ya que se le agrega un porcentaje de finos en el tamiz $\mathrm{N}^{\circ} 30$ y N 100 , como se muestra en la figura 2. Además el porcentaje de reemplazo $40 \%$ tiene antecedentes de buen comportamiento y desempeño en hormigones [1], y es justamente este porcentaje de reemplazo el utilizado en este estudio.

La granulometría del árido combinado logra ser la ideal de los áridos estudiados, por lo que su utilización se considera aceptable y adecuada para la confección de morteros de pega, tomando en consideración que es mejor que la de la arena Biobío. 


\section{Dosificación}

Se utilizaron los fundamentos establecidos en el Manual del Mortero del Instituto Chileno del Cemento y el Hormigón de Chile [9], respecto a la proporción por peso y a la corrección por humedad. Se confeccionaron dos coladas de mortero, uno llamado Mortero Patrón (MP) con 0\% de EFC y otro Mortero con Escoria (ME) con 40\% de EFC, ambos con razón A/C igual a 0.6 y relación c/a igual a 1:3. En el cuadro 2 se presenta un resumen de la dosificación empleada en la fabricación de las coladas de mortero.

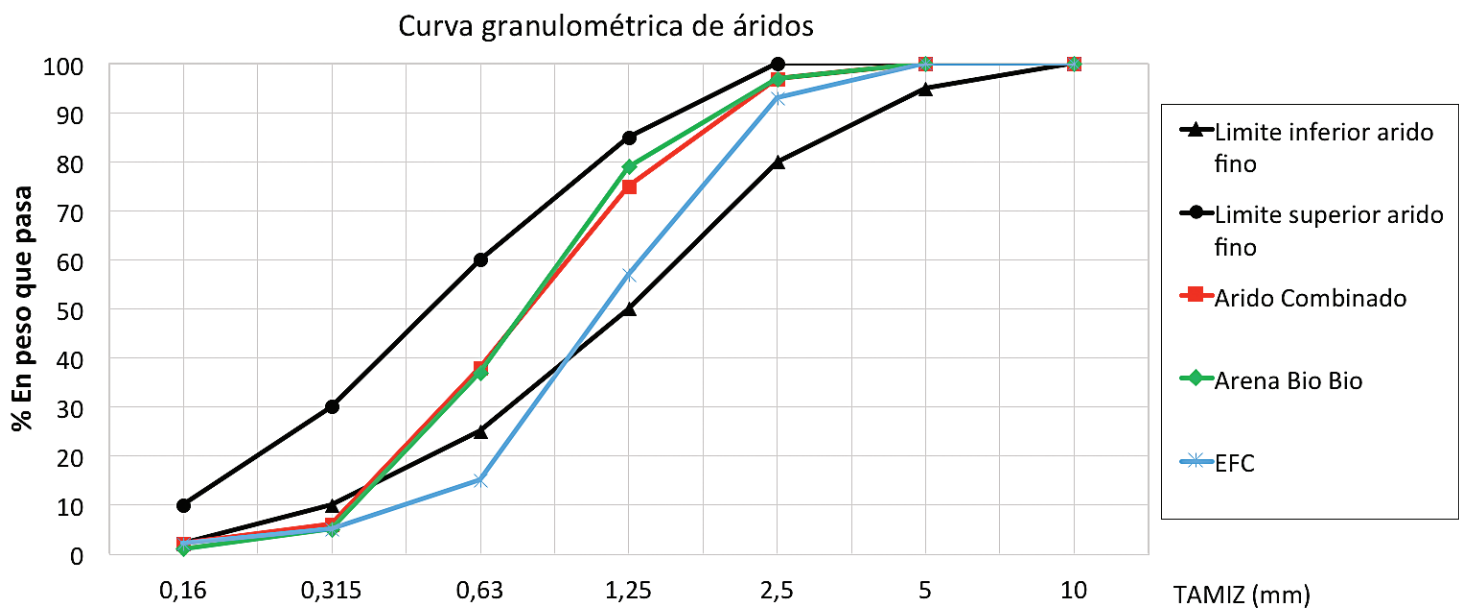

Figura 2. Curva granulométrica de los áridos finos utilizados.

Cuadro 2. Dosificación en peso de coladas de mortero [1].

\begin{tabular}{|c|c|c|c|c|}
\hline & \multicolumn{2}{|c|}{ 0\% EFC } & \multicolumn{2}{c|}{$40 \%$ EFC } \\
\hline Material & Peso $(\mathrm{kg})$ & Volumen $(\mathrm{I} / \mathrm{m} 3)$ & Peso $(\mathrm{kg})$ & Volumen $(\mathrm{I} / \mathrm{m} 3)$ \\
\hline Cemento & 475 & 169 & 475 & 169 \\
\hline Agua & 285 & 285 & 285 & 285 \\
\hline Aire & - & 30 & - & 30 \\
\hline EFC & 0 & 0 & 784,3 & 206,4 \\
\hline Arena & 1393,1 & 516 & 835,9 & 309,6 \\
\hline Total & 2153,1 & 1000 & 2380,1 & 1000 \\
\hline
\end{tabular}

\section{Metodología}

Para lograr el objetivo es necesario caracterizar experimentalmente el comportamiento mecánico de moteros de pega MP y ME. Para esto se realizaron ensayos normativos de consistencia, densidad, flexión y compresión para medir las propiedades del mortero de pega. Además de ensayos de resistencia de adherencia, resistencia básica a la compresión y al corte para evaluar la influencia de MP y ME en las capacidades resistentes de muros de albañilería. En todos los casos se realizaron al menos las repeticiones mínimas requeridas por norma para cada tipo de ensayo. De esta manera, para cada colada de mortero (MP y ME) se 
confeccionaron 9 probetas RILEM (3 para ser ensayas a los 7 días y 6 para ser ensayadas a los 28 días) con tal de evaluar sus resistencias mecánicas y densidad, 6 probetas de adherencia para evaluar la resistencia de adherencia, 5 prismas de albañilería para evaluar la resistencia básica a la compresión, y por último se confeccionaron 5 muretes de albañilería para evaluar la resistencia básica al corte. Cabe destacar además que para todas las probetas confeccionadas se evaluó la consistencia del mortero.

- Ensayo flexión y compresión: Siguiendo las indicaciones de NCh 158 Of. 67 [10], se aplica la carga vertical en el centro de la probeta RILEM $(4 \times 4 \times 16 \mathrm{~cm})$ a una velocidad constante, luego los trozos son ensayados a la compresión a una velocidad constante. Las probetas una vez confeccionadas son sumergidas en un recipiente saturado con cal, hasta el día del ensayo (7 o 28).

- Ensayo de adherencia a cizalle: Siguiendo las indicaciones de NCh 167 Of. 2001 [11], se aplica la carga vertical a una velocidad constante de modo de someter la junta del mortero a un esfuerzo de corte, producto del desnivel provocado por los refrentados confeccionados en las caras de apoyo (mezcla yeso-cemento proporción 1:1 en peso) (figura 3). En el proceso de curado las probetas se mantienen 7 días cubiertas con polietileno para posteriormente mantenerse en las condiciones ambientales de laboratorio hasta el día 28 del ensayo.
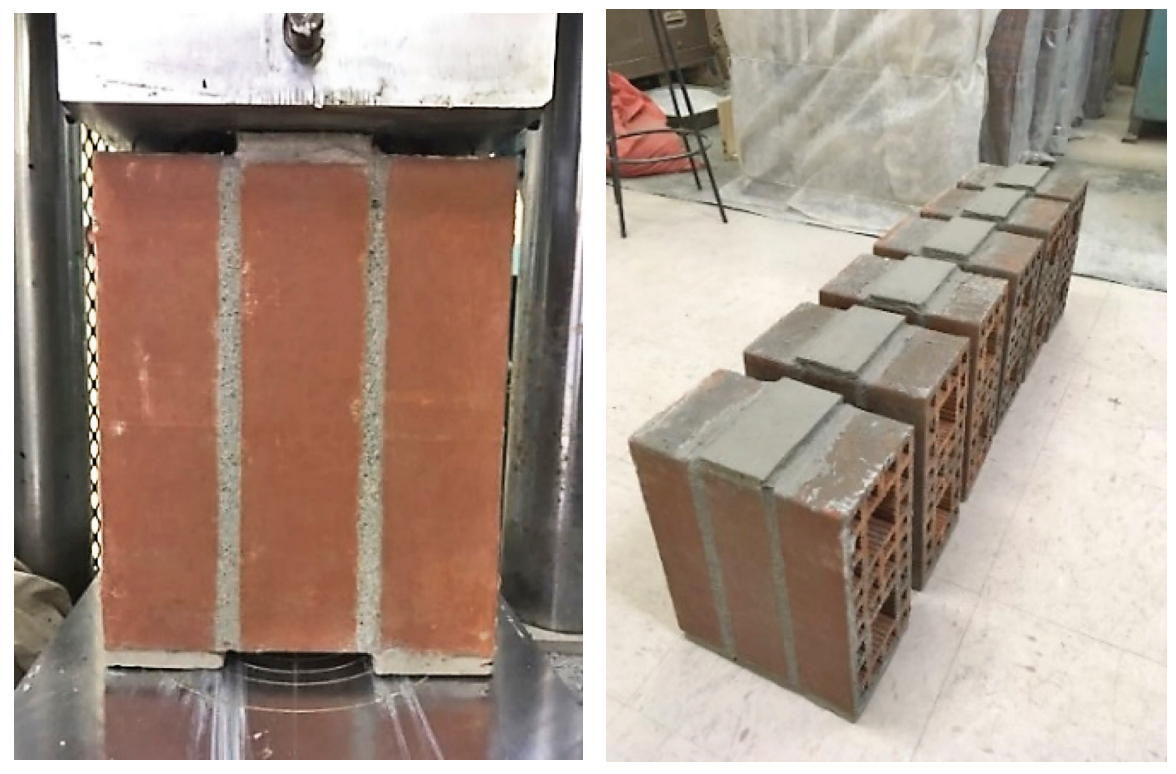

Figura 3. Esquema del ensayo de adherencia a cizalle.

- Ensayo de prismas de albañilería: Siguiendo las indicaciones de NCh 1928 Of. 2003 [12], se aplica la carga vertical a una velocidad uniforme. Se refrentaron sus extremos con una pasta de yeso de 3,5 mm (figura 4). En el proceso de curado las probetas se mantienen 14 días cubiertas con polietileno para posteriormente mantenerse en las condiciones ambientales de laboratorio hasta el día 28 del ensayo.

- Ensayo de muretes de albañilería: Siguiendo las indicaciones de NCh 2123 Of. 2003 [13], se aplica la carga vertical en forma diagonal a una velocidad uniforme mediante unos cabezales de apoyo. Se refrentaron sus vértices de apoyo con una pasta de yesocemento en proporción 1:1 en peso de $4 \mathrm{~mm}$ (figura 5). En el curado las probetas se mantienen 14 días cubiertas con polietileno para posteriormente mantenerse en las 
condiciones ambientales hasta el día 28 del ensayo.
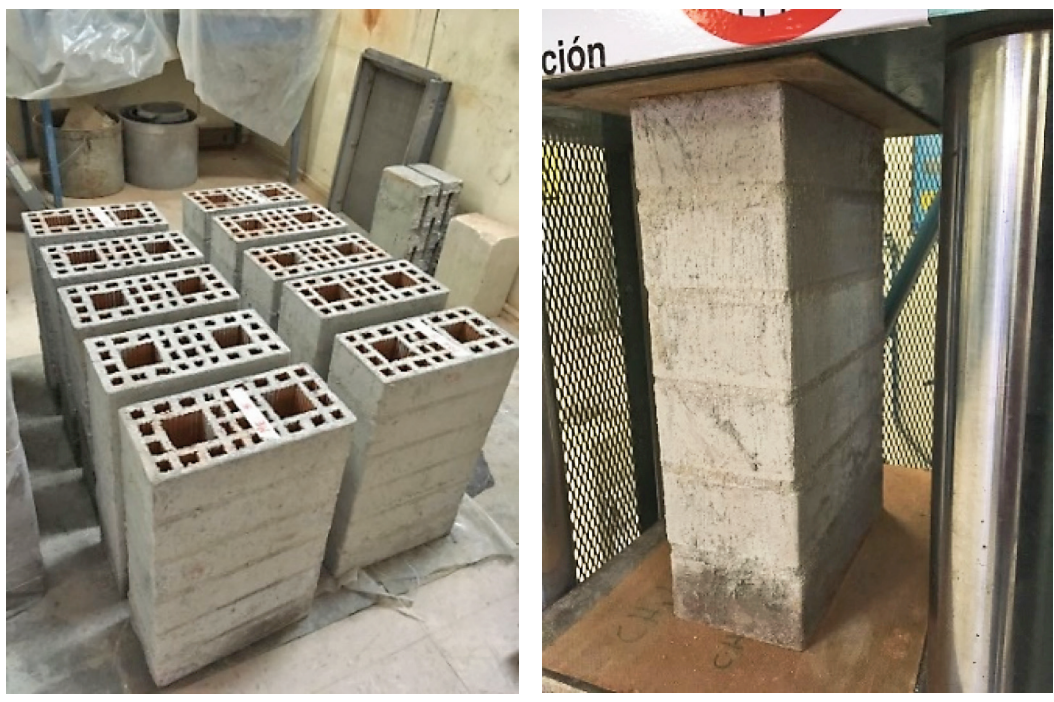

Figura 4. Esquema del ensayo de prismas de albañilería.
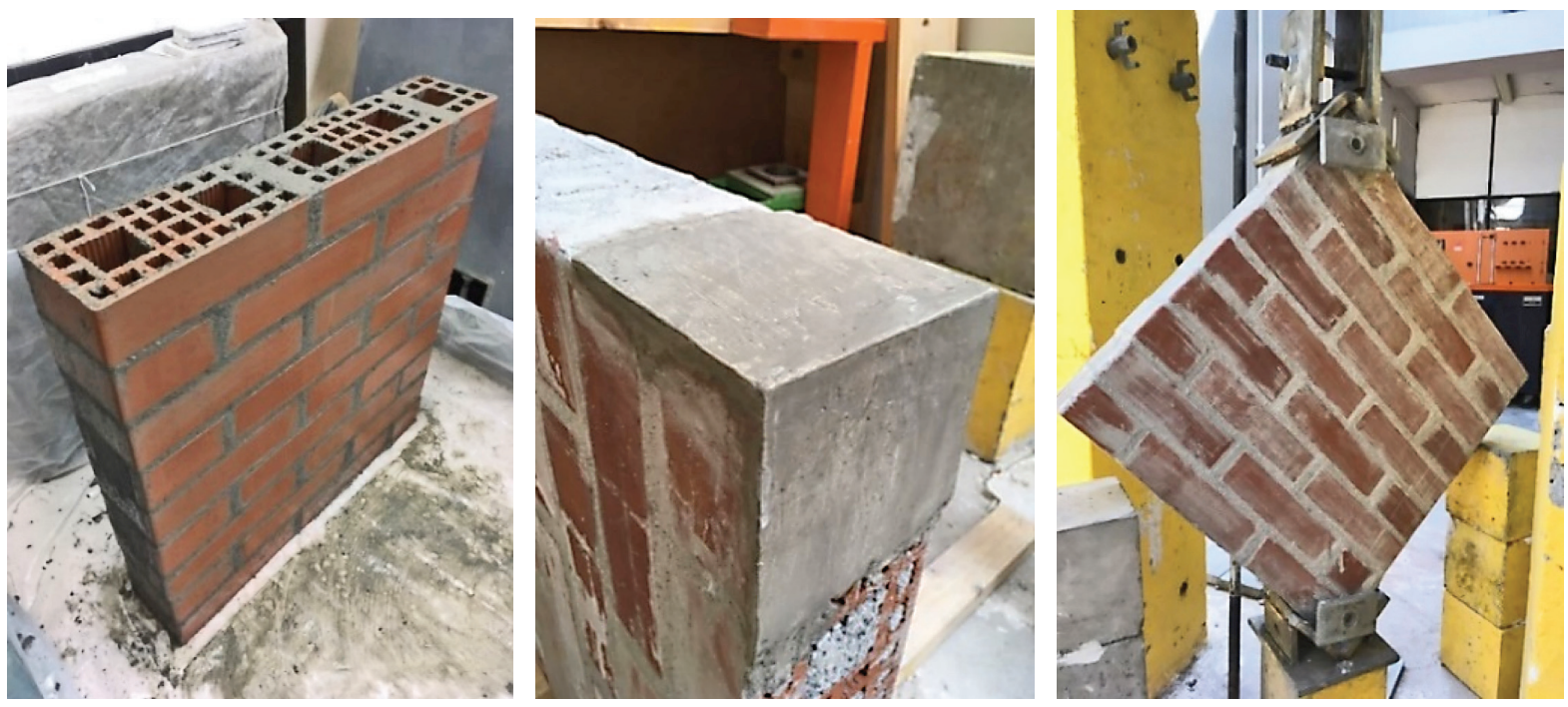

Figura 5. Esquema del ensayo de muretes de albañilería.

\section{Resultados}

Consistencia: En el mortero en estado fresco se mide la consistencia a través del cono reducido según lo indicado en la NCh 2257 Of. 96 [14]. Los valores medios de cada colada se grafican en la figura 6 donde se observa un incremento de la consistencia del mortero con EFC respecto al mortero patrón, debido a que esta se ve influenciada por la textura lisa y poco porosa, la baja absorción y la forma granular-cúbica que poseen las EFC.

Densidad: En el mortero endurecido se mide la densidad media de acuerdo con la NCh 158 Of. 67. Los valores obtenidos se presentan gráficamente en la figura 7. Se observa que la 
sustitución de arena por EFC genera un incremento en la densidad, alcanzando un 10,2\% de incremento. Esto se atribuye al alto peso específico que presentan las EFC (40\% mayor al de la arena Bío-Bío), produciendo un aumento en la densidad media del mortero.

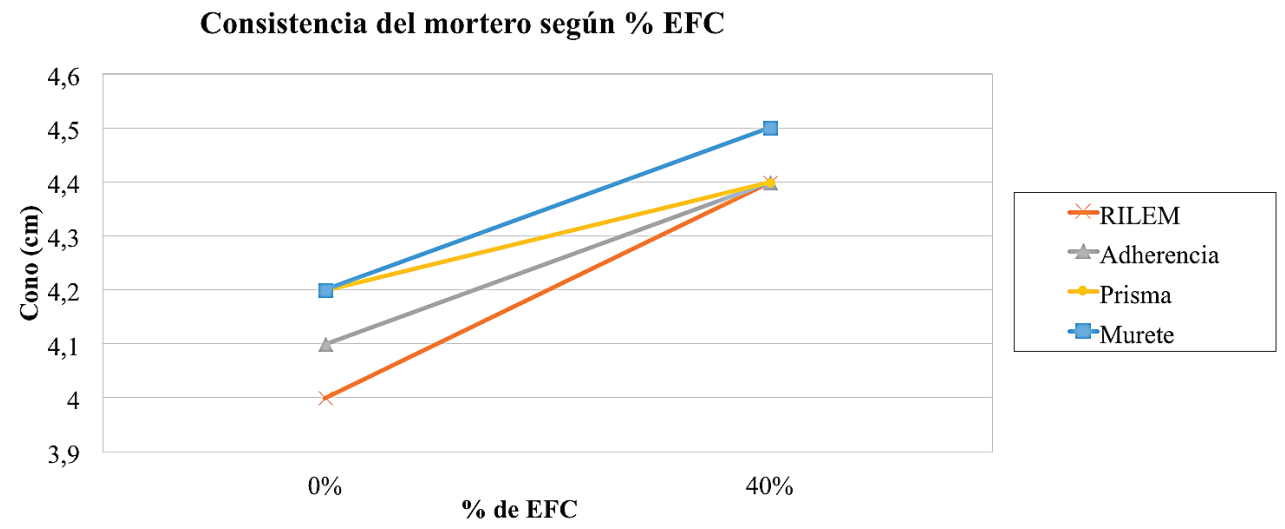

Figura 6. Variación de la consistencia por probetas, en función del contenido de EFC.

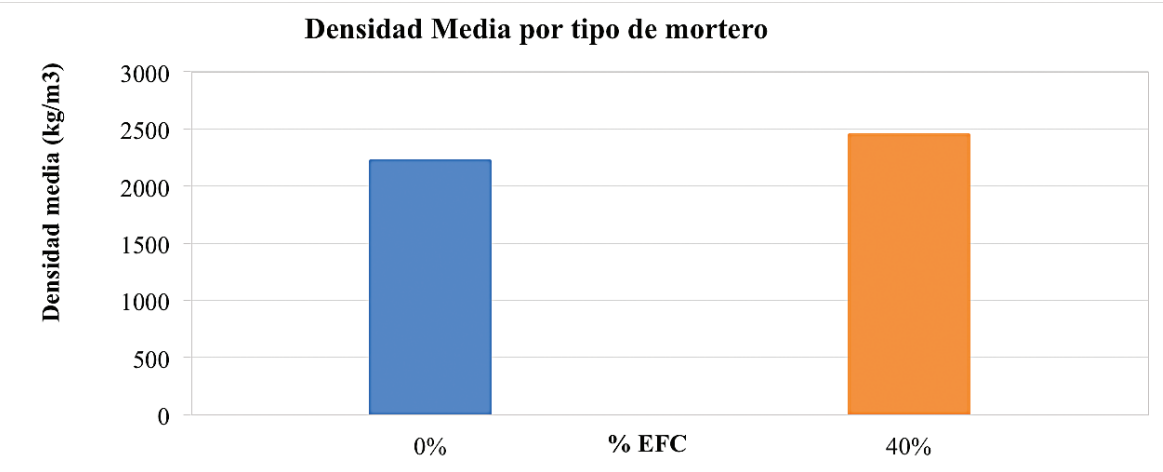

Figura 7. Variación de la densidad en función del contenido de EFC.

Resistencia a la flexión: En el mortero endurecido se mide la resistencia a flexión de acuerdo con la NCh 158 Of. 67. Los valores medios de las coladas de mortero se grafican en la figura 8.

Resistencia a la flexión de mortero

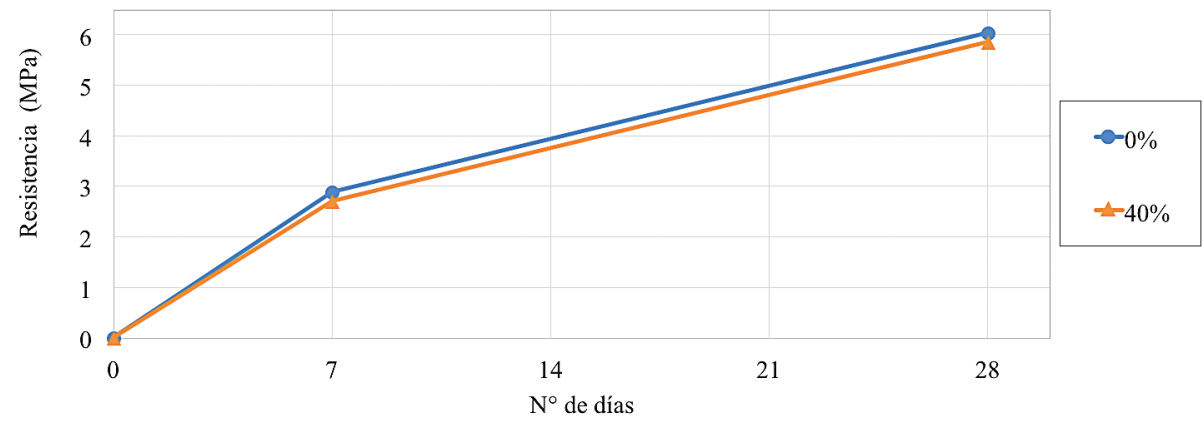

Figura 8. Resistencia a la flexión en función del contenido de EFC. 
Se observa que el mortero con $40 \%$ de EFC posee una leve disminución de resistencia a la flexión con respecto al mortero patrón para los 7 y 28 días, ya que existe una variación del 7\% y $3 \%$ respectivamente. Esta disminución se considera despreciable ya que está dentro de los márgenes de errores experimentales. Se concluye entonces que la adición de EFC mantiene la resistencia a flexión en morteros de pega para un porcentaje de reemplazo del 40\%.

Resistencia a la compresión: En el mortero endurecido se mide la resistencia a compresión de acuerdo con la NCh 158 Of. 67. Los valores medios de mortero se grafican en la figura 9.

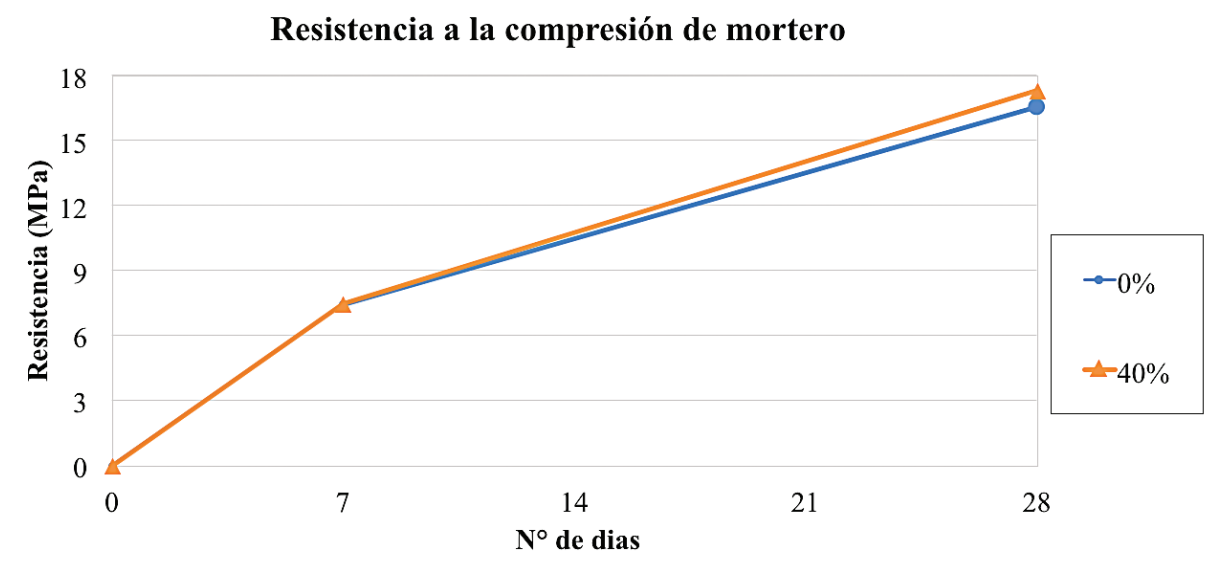

Figura 9. Resistencia a la compresión en función del contenido de EFC.

Se observa que el mortero con $40 \%$ de EFC posee un leve incremento de resistencia a la compresión con respecto al mortero patrón para los 7 y 28 días, ya que existe una variación del $0,3 \%$ y $4 \%$ respectivamente. Este incremento se considera despreciable ya que está dentro de los márgenes de errores experimentales. Se concluye entonces que la adición de EFC mantiene la resistencia a compresión en morteros de pega para un porcentaje de reemplazo del 40\%.

Resistencia de adherencia a cizalle: En el mortero endurecido se mide la resistencia de adherencia de acuerdo con la NCh 167 Of. 2001. Los valores medios de las probetas se grafican en la figura 10. Se puede destacar que el mortero con 40\% de EFC tiene un incremento de resistencia del $20,2 \%$ con respecto al mortero patrón, por lo que la EFC influye significativamente en la resistencia de adherencia del mortero de pega.

Resistencia básica a la compresión: en el mortero endurecido se mide la resistencia básica de compresión de acuerdo con la NCh 1928 Of. 2003. Los valores medios de los prismas ensayados se grafican en la figura 11. De acuerdo con los resultados observados se puede destacar que el mortero con $40 \%$ de EFC tiene un incremento de resistencia del 18,1\% con respecto al mortero patrón, por lo que la EFC influye significativamente en la resistencia básica a la compresión de prismas de albañilería. 


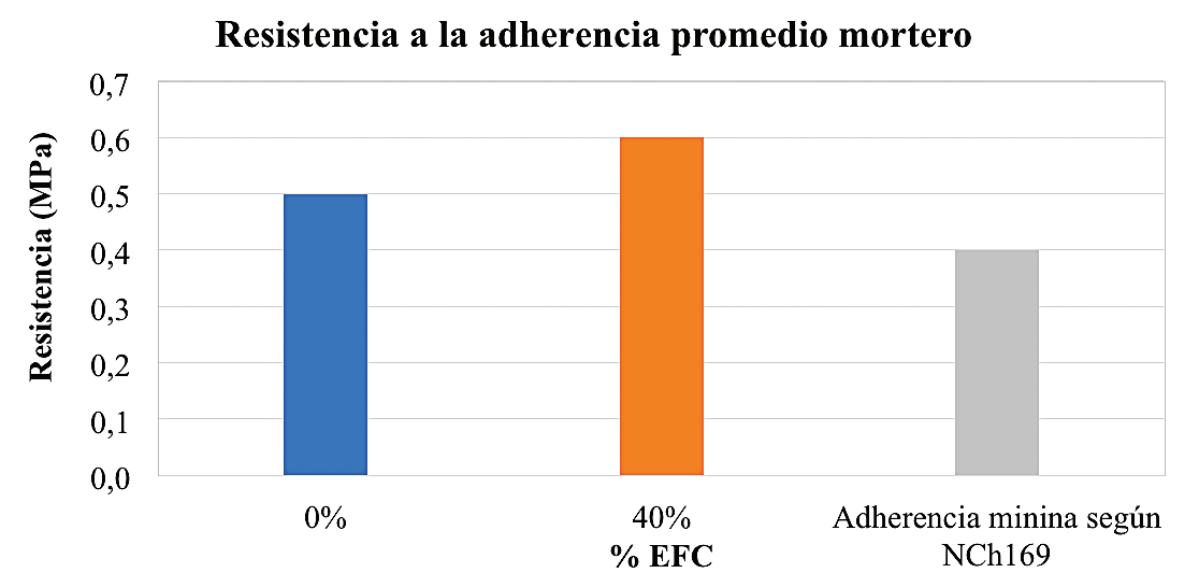

Figura 10. Resistencia a la adherencia en función del contenido de EFC.

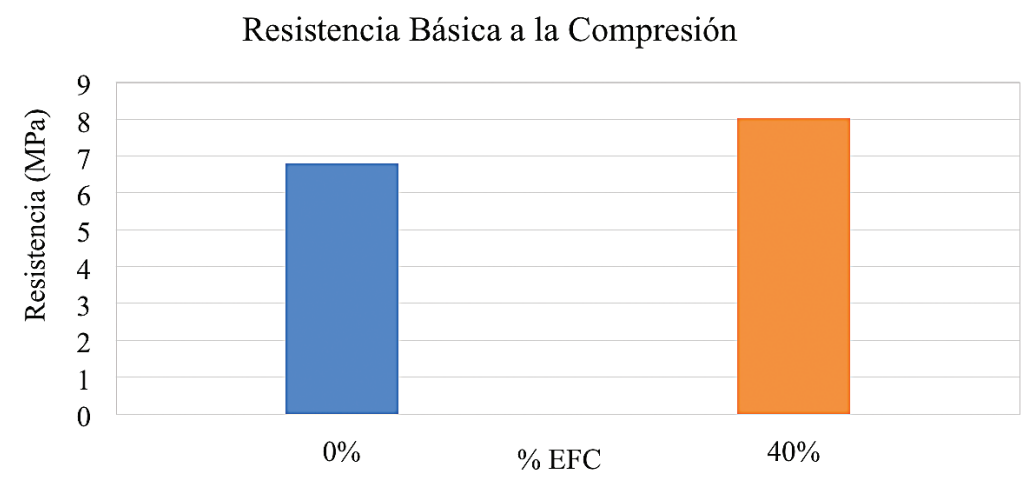

Figura 11. Resistencia básica a la compresión en función del contenido de EFC.

Resistencia básica de corte: En el mortero endurecido se mide la resistencia básica de corte de acuerdo con NCh 2123 Of. 2003. Los valores medios de los muretes ensayados se grafican en la figura 12. Se destaca que el mortero con $40 \%$ de EFC tiene un incremento de resistencia del $12 \%$ con respecto al mortero patrón, por lo que la EFC influye significativamente en la resistencia básica al corte de los muretes de albañilería. 


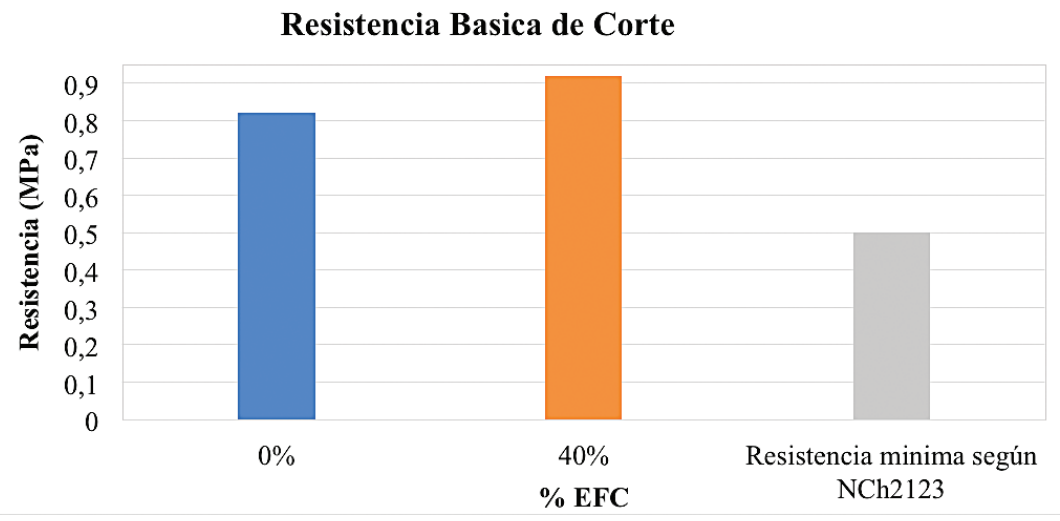

Figura 12. Resistencia básica al corte en función del contenido de EFC.

Modos de falla muretes de albañilería: Para los muretes confeccionados con mortero ME se tiene como mecanismo de falla predominante la tracción diagonal, que se caracteriza por tener un patrón de agrietamiento diagonal de forma monolítica. Sin embargo para los muretes confeccionados con mortero MP se produjeron dos fallas combinadas de adherencia y tracción diagonal (figura 13) en los muretes que soportaron la menor carga de compresión diagonal. Esto debido a que el mortero patrón tiene menor resistencia a la adherencia.
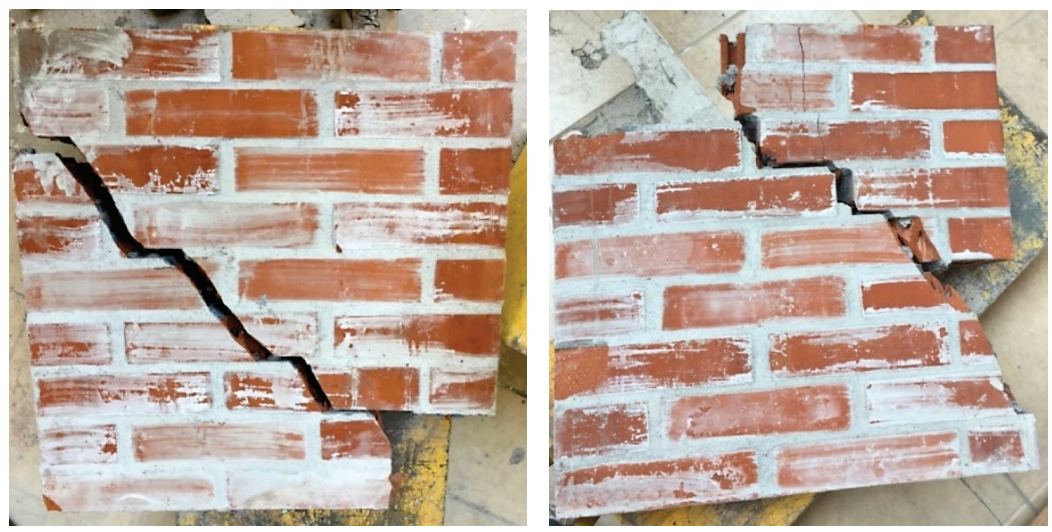

Figura 13. Modos de falla observados, falla por tracción diagonal (Izq.) y falla combinada (Der.).

\section{Conclusiones y recomendaciones}

Dentro de las principales conclusiones obtenidas en el marco de la presente investigación, se destacan:

1. La incorporación de EFC mejora la granulometría de la mezcla debido al aumento del contenido de finos.

2. Se observa un leve incremento de la consistencia de los morteros que contienen EFC respecto al mortero patrón. Esto se atribuye a la forma, textura y absorción de las EFC.

3. En el mortero endurecido se observa que la sustitución de arena por el $40 \%$ de EFC genera un incremento sustancial en la densidad del mortero, alcanzando un 10,2\% de incremento. Lo anterior se atribuye al alto peso específico que presenta la escoria, la cual 
genera un aumento de la densidad media.

4. Las resistencias a flexión y compresión de los morteros de pega se mantienen invariables para un porcentaje de reemplazo del $40 \%$ de EFC, debido a que los porcentajes de variación de las resistencias entre MP y ME son despreciables.

5. La resistencia a la adherencia, básica a la compresión y básica al corte aumentaron significativamente con la adición de la EFC, respecto al mortero patrón. Esto se atribuye principalmente a la similitud de su composición química con el cemento, logrando un mayor poder cementante en la mezcla.

6. Es factible la utilización de morteros de pega con EFC en muros de albañilería confinada (40\% de reemplazo, [1]), ya que el uso de la EFC influye significativamente en las propiedades mecánicas de estos. Por lo que, la EFC es una buena alternativa para ser utilizada como árido fino de morteros de pega sustentables.

7. Se recomienda evaluar morteros con distintos porcentajes de reemplazo de EFC y en otras aplicaciones, debido a los buenos resultados obtenidos en la presente investigación.

\section{Referencias}

[1] Cendoya, P. "Efecto en la resistencia de las escorias de fundición de cobre como agregado fino en el comportamiento resistente del hormigón". Revista Chilena de Ingeniería. Vol. 17 №1, pp. 85-94, 2009.

[2] W. Moura and D. Coutinho. "Influence of copper slag admixture in concrete in durability properties". Ambiente Construido. Porto Alegre. Vol. 4 N² 2, pp. 41-56, April 2004.

[3] Hwang CL, Laiw JC. "Properties of concrete using copper slag as a substitute for fine aggregate". Proceedings of the 3rd international conference on fly ash, silica fume, slag, and natural pozzolans in concrete, SP-114-82, pp. 1677-1695, 1989.

[4] Li F. "Test research on copper slag, concrete". Journal of Fuzhou University (Natural Sciencie Edition), Vol. 127 $N^{\circ}$ 5, pp. 59-62, 1999.

[5] Zong L. "The replacement of granulated copper slag for sand concrete". Journal of Qingdao Institute of Architecture and Engineering, Vol. $24 \mathrm{~N}^{\circ}$ 2, pp. 20-22, 2003.

[6] K. Al-Jabri, R. Taha and M. Al-Ghassani. "Use of copper slag and cement by-pass dust as cementitious materials". Cement Concrete Aggr, Vol. 24 № 1, pp. 7-12, 2002.

[7] L. Cares. "Analisis teorico-experimental de la resistencia a la flexotraccion de hormigones con escoria de fundición de cobre". Tesis para optar al título de Ingeniero Civil. Departamento de Ingeniería Civil. Universidad de Concepción. Chile, 2007.

[8] NCh 163 Of. 79. "Aridos para morteros y hormigones - Requisitos generales". Instituto Nacional de Normalización. Chile.

[9] H. Zabaleta y J. Egaña. "Manual del mortero". Instituto del Cemento y del Hormigon en Chile. Chile, 1988.

[10] NCh 158 Of. 67. "Cementos - Ensayo de flexión y compresión de morteros de cemento". Instituto Nacional de Normalización. Chile.

[11] NCh 167 Of. 2001. "Construcción - Ladrillos cerámicos - Ensayos". Instituto Nacional de Normalización. Chile.

[12] NCh 1928 Of. 2003. "Albañileria armada - Requisitos para el diseño y cálculo". Instituto Nacional de Normalización. Chile.

[13] NCh 2123 Of. 2003. "Albañilería Confinada: Requisitos de diseño y cálculo". Instituto Nacional de Normalización. Chile.

[14] NCh 2257/1 Of. 96. "Morteros - Determinación de la consistencia - Parte 1: Método del extendido en la mesa de sacudidas". Instituto Nacional de Normalización. Chile. 